\title{
Cuenta de alto costo como fuente primaria para establecer perfiles epidemiológicos de pacientes adultos con leucemias atendidos en la Clínica Valle del Lili entre 2015 y 2017
}

\author{
- Elizabeth Arrieta, Joaquín Rosales, Juan Guillermo Restrepo, Yairayobana Gil
}

Fundación Valle del Lili (Cali, Valle del Cauca)

Introducción: la cuenta de alto costo (CAC) es un organismo del sistema de salud de Colombia que obliga a las EPS de ambos regímenes a asociarse para abordar el alto costo y estandarizar las mediciones de los desenlaces de las enfermedades de alto costo, entre ellas las leucemias. En nuestro centro se hace registro prospectivo de los datos de la CAC; el objetivo de este trabajo es evaluar si los datos de la CAC pueden usarse para levantar el perfil epidemiológico de leucemias en pacientes adultos manejados en la Fundación Valle del Lili.

Materiales y métodos: estudio de corte transversal, basado en el registro prospectivo de la CAC institucional en la Fundación Valle del Lili, entre 2015 y 2017, usando variables clínicas y demográficas reportadas calculando estadísticas descriptivas de cada variable procesadas con el software Stata 11.

Resultados: se reportó a la CAC en el año 2017 un total de 592 leucemias, en pacientes adultos y pediátricos; al excluir la población pediátrica, se reportaron 316 pacientes adultos con leucemia, de los cuales 170 fueron diagnosticados entre 2015 y 2017, edad promedio 46,5 años ( \pm de 21), edad máxima al diagnóstico 92 años, el 57\% de género masculino, el 74\% del régimen contributivo, el $6 \%$ de los pacientes tenían diagnóstico previo de otra neoplasia, el tiempo medio entre el ingreso a la institución y diagnóstico es de 1 día. De las leucemias reportadas, el 51,7\% eran de estirpe linfoides y el 63,53\%, leucemias agudas, siendo las leucemias linfoblásticas agudas el diagnóstico más frecuente. El 76,5\% de los pacientes con leucemias reportadas estaban vivos hasta la fecha del registro; de las leucemias agudas, el $67,6 \%$ están vivos y, de las leucemias crónicas, el $92 \%$.

Conclusiones: los datos de obligatoria notificación a la cuenta de alto costo pueden servir localmente para el planteamiento del perfil epidemiológico de las leucemias agudas en nuestro centro y zona geográfica. 